\title{
Evaluation of Thermal Fatigue Life of Solder Joints in Electronic Devices Based on Stress and Strain Singularity Parameters
}

\author{
Jihong Liu* \\ * Technology and Innovation Center (TIC) \\ Daikin Industries, Ltd. \\ 1-1 Nishi-Hitotsuya, Settsu, Osaka 566-8585, Japan \\ jihong.liu@daikin.co.jp, web page: https://www.daikin.com
}

\begin{abstract}
It is generally known that cracks are likely to occur in the vicinity of the solder joints due to repeated temperature changes over a long time, which greatly affect the thermal fatigue life of electronic devices. Currently, the thermal fatigue life of solder joints in the electronic devices is evaluated mainly by test methods such as thermal cycle test and power cycle test, but in order to improve the efficiency and completeness of development, it is strongly desired to evaluate the thermal fatigue life of solder joints using analytical methods.

Until now, several studies on the analytical methods of evaluating the thermal fatigue life of solder joints in electronic devices have been conducted [1-4]. In these studies, in order to avoid the singularity of stress and strain fields near the solder joint edges due to dissimilar material bonding, the thermal fatigue life of solder joints is evaluated using the maximum stress or strain amplitude of a representative point near the solder joint edges. These methods are useful and practical when the solder joints have similar structures and the same calculation accuracy is ensured for stress or strain. However, it is not suitable for generalization because the selection of the representative point involves arbitrariness and trial and error.

Since different materials are combined, the stress and strain fields near the solder joints have singularity. In generally, the behaviours of the singular stress and strain fields can be completely described by the singularity orders and the singularity strength coefficients. Therefore, in this study, we tried to evaluate the thermal fatigue life of the solder joints using the stress and strain singularity parameters near the solder joint edges. Specifically, firstly, the material properties of the solders were acquired using the material tests and their creep constitutive equations are constructed. Next, the stress and strain singularity parameters in the vicinity of the solder joints for power modules were calculated under the thermal cycle test conditions using finite element method (FEM) analyses. Finally, the stress and strain singularity parameters and the fatigue test results are associated with each other by fatigue laws, and the evaluation equations for predicting the thermal fatigue life of the solder joints are constructed and their accuracy are confirmed reasonably well. It is clarified that the fatigue laws based on the stress and strain singularity parameters are valid and useful for predicting the thermal fatigue life of solider joints in electronic devices.
\end{abstract}

\section{REFERENCES}

[1] Z. Guo and H. Gonrad, "Fatigue crack growth rate in $63 \mathrm{Sn} 37 \mathrm{~Pb}$ solder joints", Transactions of ASME, Journal of Electronic Packaging (1993) 115:159-164.

[2] M. Mukai, T. Kawakami, Y. Hiruta, K. Takahashi, K. Kishimoto and T. Shibuya, "Fatigue life estimation for solder joints in SMT-PGA packages", Transactions of the ASME, Journal of Electronic Packaging (1998) 120:207-212.

[3] H. Takahashi, T. Kawakami, M. Mukai and N. Ohno, "Thermal fatigue life simulation for Sn$\mathrm{Ag}-\mathrm{Cu}$ lead-free solder joints", Journal of the Japan Institute of Electronics Packaging (2004) 7:308-313.

[4] T. Terasaki, H. Tanie, N. Chiwata, M. Wakano and M. Fujiyoshi, "Use of modified accumulated damage model to predict fatigue failure lives of $\mathrm{Sn}-\mathrm{Ag}$-Cu-based solder joints in ball-grid-arraytype packages", Transactions of the Japan Institute of Electronics Packaging (2012) 5:1-11. 